\title{
Hand in Air Tapping: a Wearable Input Technology to Type Wireless*
}

\author{
Leonardo Meli ${ }^{1}$, Davide Barcelli ${ }^{1}$, Tommaso Lisini Baldi ${ }^{1,2}$, and Domenico Prattichizzo ${ }^{1,2}$
}

\begin{abstract}
We present Hand in Air Tapping (HAT), a wearable input interface which allows interactions through fingers tapping. It consists in a Bluetooth Low Energy rings enabling wireless communication with any compatible device. Each ring is hardware-wise independent of the others. This allows full modularity, i.e., the number of employed devices can be chosen to meet each application requirements. The proposed system was evaluated in two user studies, both on text input: (1) users learning curve in terms of writing speed; (2) rate of text entry comparison between the proposed interface and that of numpad style keyboards. We associated each keystroke to a set of letters/symbols and compared two approaches: one based on T9 technique and the other on multi-tap input method. Results show comparable performance between HAT and numpad style keyboards. HAT keeps the hands free, not affecting hand movements and human interactions with the surroundings. Moreover, as a general input technology, it might have several potential applications in the field of computer-human interfaces.
\end{abstract}

\section{INTRODUCTION}

Typing technologies have come a long way over the past couple of centuries. Among them, typewriters have totally changed their shape over the years: they have been updated in terms of technology, efficiency, design and eventually they became keyboards in the age of computers. However, people still have something with letters in front of them. Although this idea, as simple as functional, has remained the same for many years, it has limitations in terms of size, weight, and portability.

The recent wide dissemination of wearable interfaces may change old habits, addressing these issues. World's major companies are highly sponsoring devices like the Apple Watch, the Google Moto 360, and the Asus ZenWatch. Wearables have the advantages of being portable and well integrated into people habits, with the goal of providing valuable information to/from the users. Technology will increasingly become a consistent part of our life as it will be part of our clothing and sometimes even part of our bodies. In the last years, there has been an increasing interest in developing new solutions to accurately track the human body and enhance human-computer interactions. Focusing on hands, several approaches have been developed to interact

*This research has received funding from the European Union Seventh Framework Programme FP7/2007-2013 under grant agreement $n^{\circ} 601165$ of the project "WEARHAP" and from the European Union's Horizon 2020 Research and Innovation Programme under Grant Agreement n'688857 of the project "SoftPro".

${ }^{1}$ Department of Information Engineering and Mathematics, University of Siena, Via Roma 56, 53100 Siena, Italy meli, barcelli, lisini, prattichizzo\}@dism.unisi.it

${ }^{2}$ Department of Advanced Robotics, Istituto Italiano di Tecnologia, Via Morego 30, 16163 Genoa, Italy

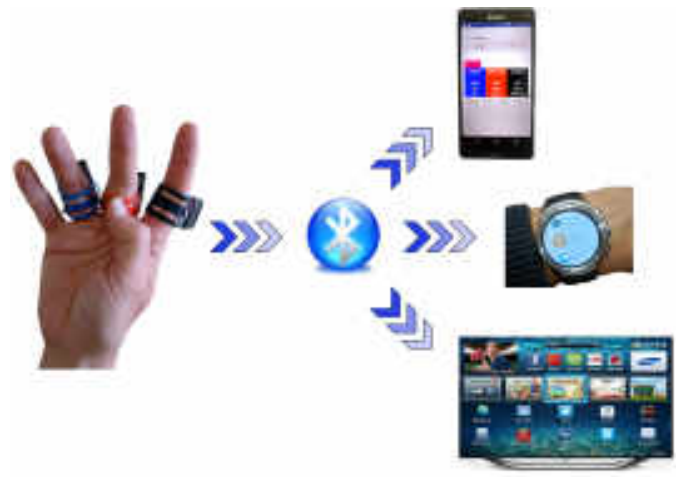

Fig. 1: HAT is a modular input interface composed of indipendent touch sensitive rings capable of enabling Bluetooth Low Energy communication with any compatible device, such as mobile phones, smartwatches, and smart TVs.

with virtual reality using wearables. Examples of these strategies include MARG [1], piezoresistive [2], magnetic, and Hall-effect sensors [3]. Besides input interfaces, wearable technologies are widely used also for providing haptic feedback. Meli et al. [4], [5] presented a wearable cutaneous haptic device able to provide cutaneous stimuli at the finger pad. Ir reproduces a virtual object by recreating the fingertip deformation.

According to eMarketer, nearly 39.5 million US adults used wearables in 2016, and this number is only going to rise. A new concept of fashion is born, that mixes advanced electronic technologies with common clothes and accessories.

\section{RELATED WORK AND CONTRIBUTION}

As interest in wearables grows, researchers explore new ways to interact with widespread electronic devices, such as smartphones, smartwatches, smart TVs, and computers in general. In 2010, the Peregrine Glove was launched to the North American PC gaming market. It consists of a glove with 38 customizable touch zones to communicate with any application that is supposed to use a keyboard. Touching your fingers can then be the way to communicate your intention. Jing et al. [6] proposed the Magic Ring, a light hands-free input device for the finger gestures recognition. It is based on inertial sensors and can act as a remote controller for electrical appliances. In 2014, a system named Airwriting aimed at translating what you write in the air into editable text for phones, or tablets, was developed at Germany's Karlsruhe Institute of Technology [7]. It uses accelerometers and gyroscopes together with a statistical model of the 
characteristic signal pattern for every alphabet letter. In 2015, Nirjon et al. [8] presented TypingRing, a wearable system that enables the user to type as if an invisible standard QWERTY keyboard is lying on a surface underneath his hand. A user study states that TypingRing yields a reasonable typing speed (e.g., 33-50 characters per minute) and the typing speed improves over time.

In recent years, we frequently use the information service coming from mobile terminals to accomplish several different tasks (e.g., checking e-mails, finding a route, texting, dialing a number). However, these hand-held mobile devices need to be taken out of a pocket to use. For example, most of the smartwatches available in the market are capable of delivering data-driven notifications and timely updates to the user, but, because of the tiny size of their screen, there is not enough space for solid gesture commands. This is why voice control is usually the primary input method for a smartwatch. Besides technological limitations due to speech recognition, for example drastically affected by the noise of the surrounding environment, also privacy issues arise. Other technologies, such as the Peregrine Glove and Airwriting, are glove-based input systems and come with all inconveniences of covering the hand. On the other hand, the Magic Ring has a very low impact in terms of obstruction, but it is unsuited for typing. To some extent, most of these devices can be used to replace a keyboard, but they are based on complex gestures that take time to master. Moreover, continuously performing gestures in uncomfortable poses and with no support for the arm can strain the user. In this regard, more recently, the Tap Strap (Tap Systems Inc., USA) came out. It has a series of embedded sensors which monitor mechanical information of the hand and fingers. Data are decoded and finger tap combinations are transformed in characters. The device must be put at the top of the fingers (near knuckles) and it involves all the five fingers. Although its very good design and its ease of donning, the user interaction with the surrounding environment is significantly constrained while wearing the device. On the other hand, TypingRing addresses the task of typing without being obstructive, but it requires a surface, such as a table or wall, to type on.

By considering all the advantages and weaknesses of the aforementioned input technologies, we propose a new system based on rings with easy typing in mind: the Hand In Air Tapping (HAT). When the thumb touches a ring, a data packet is sent via Bluetooth to a different device (see Fig. 1). We believe that devices that collect information solely relying on accelerometers, no matter the quality of the filtering process, nor the quality of the artificial recognition algorithms, suffer from user's undesired actions, especially when the hand is involved. Our main goal is to provide an unobstructive system reliable at any moment, regardless if you are sitting at your desk, or walking in the street. In the era of portable technologies we cannot constrain the use of a device to a very specific scenario only. Moreover, the number of misunderstood actions must be minimized, possibly even fully eliminated, so as to avoid turning the system from an useful tool into an annoying device.

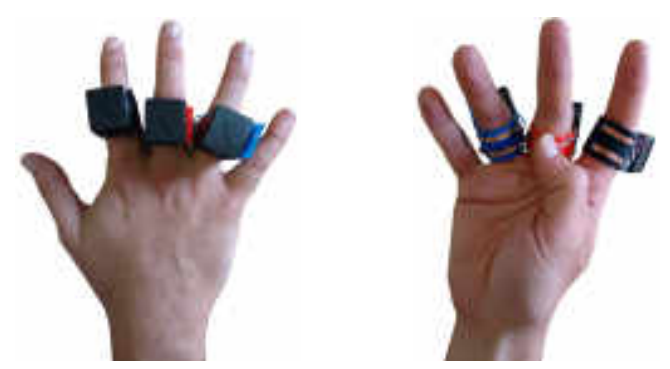

Fig. 2: Design of HAT.

\section{HAT - HAND IN AIR TAPPING SYSTEM}

\section{A. Hardware}

A 3D printed ring-shaped housing, made of ABS material, guarantees high standards of wearability and embeds the electronic core of each device. As it is shown in Fig. 2, the input interface is firmly attached on the proximal phalanx of the finger by means of an adjustable velcro strap. On it two conductive copper bands are sewn allowing 3 distinct types of contact detection: upper band, lower band and both bands simultaneously. This latter modality is achieved softwarewise by means of a timer set to $30 \mathrm{~ms}$. HAT is managed by a RFduino, a fingertip sized, Arduino compatible, wireless microcontroller (RFduino, USA). The device embeds a triple axis accelerometer ADXL362 (AnalogDevices, USA) to detect the hand tilt, thus enabling wrist movements to control the keystroke input in combination with the touch actions. Therefore, the number of available input activations for each ring is doubled, and allows to cover the whole set of required inputs using few devices. Moreover, the accelerometer set on motion-triggered wake-up mode permits to turn on the device only when needed, thus improving battery life. Each ring is powered by a tiny $(19 \times 15 \times 7.5 \mathrm{~mm}) 3.7 \mathrm{~V} 100 \mathrm{mAh}$ LiPo battery, that guarantees a relatively long lifetime (more than two days). The stand-alone hardware structure makes the system modular: according to the task to accomplish, a different number of rings (i.e., input devices) can be used.

As input strategy, we decided to avoid standard switches and push buttons, because they are heavy, arduous to wear, and characterized by a limited working frequency due to their mechanical inertia. In our opinion, the use of a high gain PNP-BJT as current amplifier, whose ground is always connected to the skin of the finger, is a more stable and robust method to detect contacts.

The total weight of each ring is $11.6 \mathrm{~g}$, including the ABS housing $(4.7 \mathrm{~g})$ and the LiPo battery $(3 \mathrm{~g})$.

\section{B. Software}

The proposed device has been evaluated in a text input case study exemplified on an Android mobile phone. In order to enforce modularity, each ring is individually programmed to exhibit a predefined behavior, i.e., notify 4 types of events: i) upper band touch detection, ii) lower band touch detection, iii) contemporary touch on both bands and $i v$ ) orientation variation. Implementing an event-triggered paradigm, each 

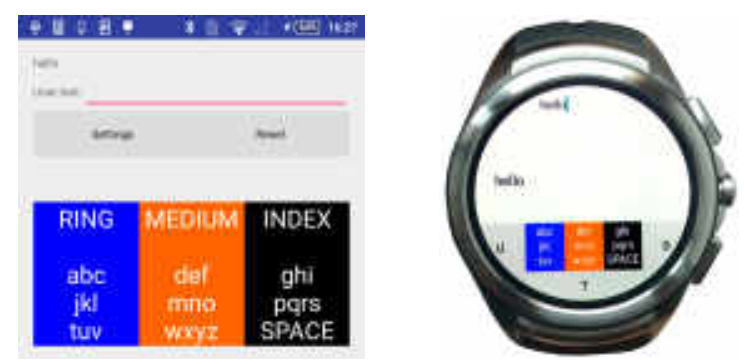

Fig. 3: Screenshots of the Android application to read signals coming from the rings running on a mobile phone and a smartwatch.

ring exploits the onboard Bluetooth Low Energy (BLE) to notify the occurred event to the phone, acting as BLE master. The binding between an event from a specific ring and a functionality is performed in the phone app and it is partially customizable to meet user's needs.

Regarding input methodologies, we implemented a multitap and a simple version of T9 [9], in which possible words for a given key combination are sorted according to their length. More sophisticated methods that consider also words usage frequency or sentence analysis will be future improvements. The app provides a simple Graphical User Interface (GUI) which displays the bindings between rings and bands with respect to the orientation of the middle finger. The positions exchange between ring and index, due to a 180 degrees hand rotation, is implemented in the GUI for easing the use. Moreover, two lines of text are included: one containing the entered text and the other displaying the word currently being typed. Finally, a settings view allows full customization in terms of ring/band and key binding.

In order to let the user benefit for a potential a priori experience with a T9 software, most likely endured by means of a numpad mobile phone, we chose to use three rings, namely index, medium, and ring, each sensing three possible touch signals plus the orientation. The total of direct key inputs is then 9 in each tilt state, resulting in a total of 18 . Following the same line, we configured the default bindings for the palm up orientation to mimic a numpad alphabetic keyboard (see Table I). We assigned all control functionalities to the palm down orientation: NEXT, PREV, SPACE, DEL, RESET, and UNDO, along with a reduced set of symbols. NEXT and PREV are used in the T9 mode to select a word from the list of those compatible with the current pressed key sequence.

In Table I, the colors of the column on the left resemble the velcro strap color of the rings. This correspondence is also kept in the GUI (see Fig. 3).

\section{EXPERIMENTAL EVALUATION}

To carachterize HAT system performance, we carried out a text input case study in which we compared it with Nokia 3310 in three experiments: (1) evaluation of operability estimation of the learning curve using a single phrase; (2) evaluation of the text entry rate while writing multiple given

\begin{tabular}{|c|c|c|c|}
\hline Palm rotation & Finger & Band & Bindings \\
\hline \multirow{9}{*}{ Upwards } & \multirow{3}{*}{ RING } & UP & $a, b, c$ \\
\hline & & UP+DOWN & $\mathrm{j}, \mathrm{k}, \mathrm{l}$ \\
\hline & & DOWN & $\mathrm{t}, \mathrm{u}, \mathrm{v}$ \\
\hline & \multirow{3}{*}{ MIDDLE } & UP & $\mathrm{d}, \mathrm{e}, \mathrm{f}$ \\
\hline & & UP+DOWN & $\mathrm{m}, \mathrm{n}, \mathrm{o}$ \\
\hline & & DOWN & $\mathrm{w}, \mathrm{x}, \mathrm{y}, \mathrm{z}$ \\
\hline & \multirow{3}{*}{ INDEX } & UP & $\mathrm{g}, \mathrm{h}, \mathrm{i}$ \\
\hline & & UP+DOWN & $\mathrm{p}, \mathrm{q}, \mathrm{r}, \mathrm{s}$ \\
\hline & & DOWN & SPACE \\
\hline \multirow{9}{*}{ Downwards } & \multirow{3}{*}{ INDEX } & UP & NEXT \\
\hline & & UP+DOWN & SPACE \\
\hline & & DOWN & PREV \\
\hline & \multirow{3}{*}{ MIDDLE } & UP & DEL \\
\hline & & UP+DOWN & \\
\hline & & DOWN & RESET \\
\hline & \multirow{3}{*}{ RING } & UP & ()[]\{\} \\
\hline & & UP+DOWN & \\
\hline & & DOWN & ,.:;?!' \\
\hline
\end{tabular}

TABLE I: Default HAT key bindings.

phrases; (3) extensive training of a single user on several different phrases.

\section{A. User Study 1 - Evaluation of operability}

This input system might appear counterintuitive and very slow to perform complex writing tasks, but, bearing in mind the average user typing speed while using numpad phones in the past years, we strongly believe that this technology is worthwhile. Learning ease is an important factor, but certainly not the only one: what matters the most for final users is the achievable efficiency and this system has a steep learning curve. Once it is mastered it will be possible to input data without looking at the rings, nor at the target device's screen. In this respect, we conducted a user study as a first step to investigate the operability. Its goal was to estimate how fast a user could improve his/her typing speed using HAT in two writing modalities: (1) using the custom T9 technique introduced in Sec. III-B; (2) using the classical multi-tap text entry modality for numpad keyboards, i.e., each time the subject touches the conductive band in a small time period (1 s), he/she switches to the next letter (refer to Table I to check the default correspondence between key bindings and conductive bands).

Participants: 7 healthy volunteers, four males and three females, took part in the experiment. They were aged between 25 and 45 (mean 33) years. All of them were righthanded. None of the participants had previous experience with HAT. All of them were aware of and had past experience with the T9 input methodology in numpad phones, but their most recent use dates back to more than three years prior to the experiment. None of them was a native English speaker.

Methods: Subjects wore three rings composing the HAT input system on the proximal phalanx of their right index, middle, and ring fingers. They were asked to slightly change the position of each device, i.e., spinning the ring, to be able to better reach all of the six conductive bands with the thumb of the same hand. An Xperia Z3 mobile 


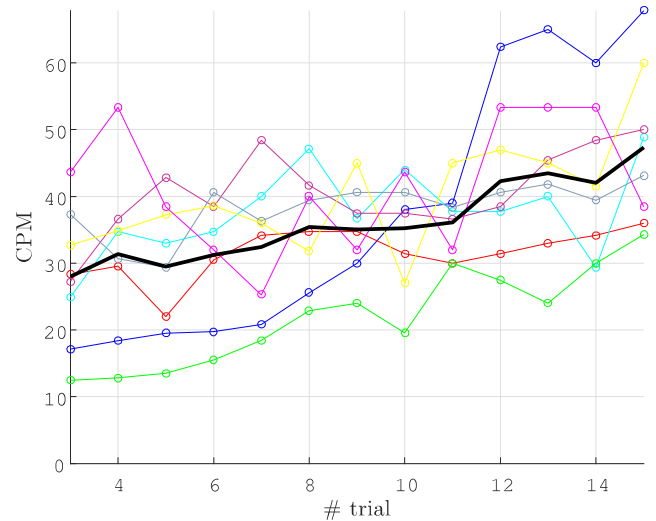

(a) Multi-tap

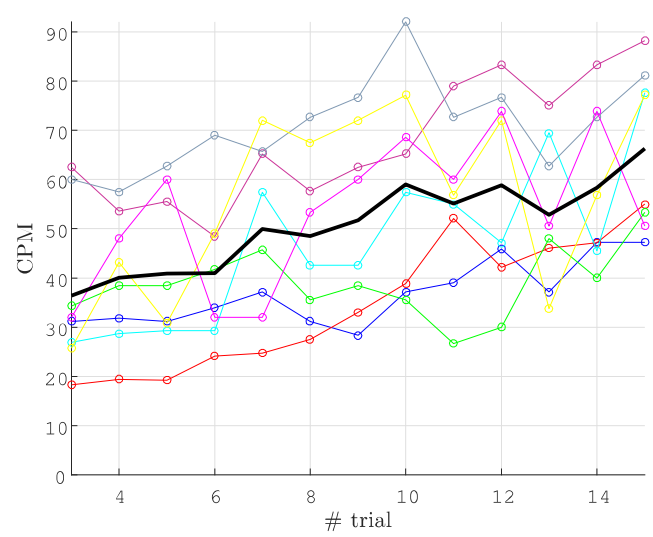

(b) Custom T9

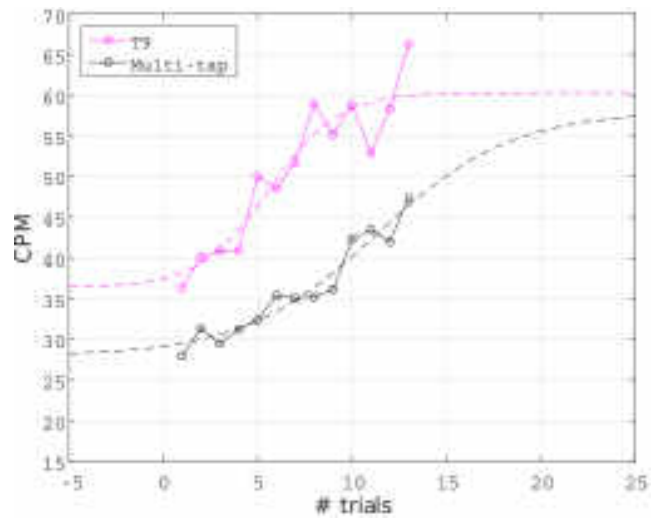

(c) HAT learning curves estimation

Fig. 4: Evaluation of operability of HAT.

(a) and (b) show the CPM about the phrase repeatedly entered throughout the experiment by each subject (one per color). The thicker black line shows the mean among all the subjects. Multi-tap and custom T9 input methods were used in (a) and (b), respectively.

(c) fitting curves (dashed lines) represents the estimation of the learning curves computed on experimental data averaged among the subjects (circles). Multi-tap and T9 techniques are depicted in magenta and black, respectively.

phone (Sony Inc., Japan) running the custom application described in Sec. III-B was used to display phrases being typed and was placed on the desk, right in front of the subjects. A familiarization period of 5 minutes was provided to participants to acquaint them with HAT. In the meanwhile they were instructed that they had to repeatedly input a single phrase (randomly selected from the MacKenzie and Soukoreff's phrase set [11]) for 15 times. This task had to be repeated using both the input conditions (i.e., multi-tap and custom T9). The presentation order of the condition was randomized and there was a break of three minutes between the two sessions, one per input modality. The merit of using a pre-defined phrase set is to attain general and repeatable results. The phrases contained no punctuation symbols, and just a few instances of uppercase characters, but participants were instructed to ignore case and to enter all characters in lowercase. Although the inclusion of such characters would make the evaluation more closely mimic real-life interactions, it would also introduce a confounding source of variation in the dependent measures, and, therefore, internal validity would be compromised and results would be less likely to attain statistical significance [11].

Results: Throughout the experiment we measured the time to complete each trial (enter the phrase once). The trial started when the user read the given phrase for the very first time and ended when that phrase was successfully typed. In case of mistakes, the user could use the DEL key to delete just the last letter, or the RESET key to start typing the whole word by scratch. We expressed the text entry rate also as characters per minute (CPM) including spaces. The first two phrases were ignored for practice and are not reported in the results plot.

Figs. $4 \mathrm{a}$ and $4 \mathrm{~b}$ show how the input speed increases trial by trial regardless the input method tested. Comparison of the means among the different trials was tested using a oneway repeated measures ANOVA for each typing modality, i.e., multi-tap and custom T9. According to Shapiro-Wilk and Mauchly's Test, the assumptions of normality and sphericity, respectively, were not violated in any case. There was a statistically significant difference for both input methods: $\mathrm{F}_{1,7}=246.336, \mathrm{p}<0.0001$, for the multi-tap method and $\mathrm{F}_{1,7}=106.711, \mathrm{p}<0.0001$, for the custom $\mathrm{T} 9$ technique.

However, it is worth to point out that the given practice is based on a single phrase and on just seven subjects, thus the obtained typing speed can serve as a rough estimation of the learning curve and of the potential limit of the typing speed using HAT.

The theory of the learning curve or experience curve is based on the simple idea that the time required to perform a task decreases as a user gains experience [12]. The use of learning curves finds application in many cases, such as setting labor standards, evaluating labor performance and productivity, preparing cost estimates, and in several other user studies [13], [14], [15], [16].

In order to assess a measure of learning, we used the sigmoid curve for fitting purposes [17]

$$
\hat{f}(\theta, x)=\frac{\theta_{4}}{\theta_{3}+e^{x \theta_{1}+\theta_{2}}}+x_{\text {min }},
$$


where $x$ is the trial number, $\theta=\left\{\theta_{i}\right\}_{i=1 \ldots 4}$ is the curve shape parameters vector and $x_{\min }$ is the minimum observed performance. We used the data plotted in Fig. 4 to feed the parameter estimation problem for both the multi-tap and T9. The resulting unconstrained minimization problem (UMP) is

$$
\theta^{*}=\arg \min _{\theta} \sum_{x=1}^{13}(y(x)-\hat{f}(\theta, x))^{2},
$$

where $x$ is the trial number and $y(x)$ is the corresponding CPM value. The dependency on the initial guess, consequence of the UMP non-convexity, was mitigated in a genetic fashion by randomizing the initial guess and storing the best solution.

Fig. $4 \mathrm{c}$ shows the fitting curves alongside the averaged experiment data. The black dashed line depicts the multitap modality for which the achieved good fitting assesses a good accuracy level for the settlement prediction of 40 CPM. The T9 converges to $60 \mathrm{CPM}$ and is shown in blue. The optimization result for this case was 5 times higher than for the multi-tap, then the multi-tap modality can be considered significantly slower. We believe that sample number 11 has an interesting interpretation: after gaining some familiarity with the device the users tried to push themselves making more mistakes and thus reducing their efficiency. That is confirmed by sample 13 in which a result greater then the predicted settlement is achieved on average. We expect that future works will provide evidence of a better converging performance of about 80 CPM.

According to subjects' feedback, a significant factor for preventing high text entry rates was the difficulty of having to look at both the phone's screen and the rings to map letters to type on bands to touch. Although participants claimed to feel the difference in terms of input speed from the first to the last phrase, they also assessed they had much room for improvement and a practice of 15 phrases is not enough to reach the best performance. A much longer practice would be needed to be proficient.

\section{B. User Study 2 - Nokia 3310 vs HAT: multi-phrase com- parison}

The goal of this study was to compare the HAT system with an as old as widespread texting method, the T9 technique implemented on a 3310 mobile phone (Nokia Ink., Finland). Because of the improvement in terms of performance when using the custom T9 technique compared to using the classical text input modality (see Fig. 4), we decided to consider only the "aided" input method, i.e., the T9 technique.

Participants: The 7 subjects who participated to the User Study 1 described earlier were again recruited for this study. For each subject this experiment took place right after the previous one. A break of 30 minutes was given to the volunteers before the start of the new experiment. It is worth to point out again that all the participants were aware of what the T9 technique was, but they had not been used it for at least three years.

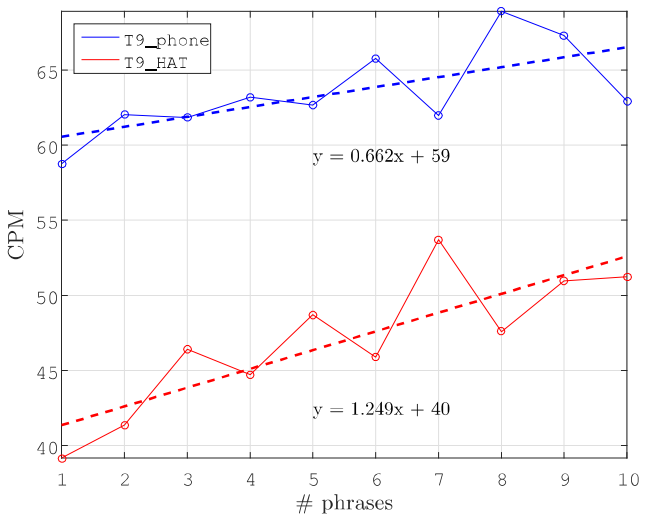

Fig. 5: Performance comparison while using the T9 technique with the 3310 mobile phone and the HAT. Solid lines show the the mean over the 5 subjects phrase by phrase. Dashed lines show the linear interpolation on the set of available data. Experimental data related to the phone and the HAT system are expressed in blue and red, respectively. The slopes of the two equations empathize the different learning effect due to the input modality tested.

Methods: The experimental setup was very similar to the one of Study 1, except that also the Nokia 3310 played the role of input device for half of the evaluation. Subjects were asked to type 10 different phrases randomly selected from the MacKenzie and Soukoreff's phrase set [11]. Each phrase had to be entered using two different modalities: (1) the HAT system with the previously described custom T9 technique and (2) the Nokia 3310 mobile phone with the T9 method enabled. Similar to Study 1, the presentation order of the input conditions for each subject was randomized and there was a break of three minutes between the two sessions. While using the mobile phone, subjects were required to use only one hand to type on the numpad keyboard. Because of the first experiment, subjects did not need to acquaint them with the HAT system. Thus, a brief familiarization period was provided to them only regarding the Nokia 3310 input modality.

Results: In order to compare the rates of text entry using the two considered input methods we recorded the time needed to successfully type each given phrase (including any time to correct errors using the DEL/RESET commands). We then transformed all the data related to time in CPM, a metric often used because it ignores the length of the words in the typing test. Spaces were considered as characters. Fig. 5 shows the performance comparison expressed in CPM between the two input methods tested: HAT in red and the phone in blue. Each circle represents the mean among all the subjects for a single phrase. It is worth emphasizing that different subjects had to always type different phrases. The two dashed lines are the linear interpolation of all the collected data relative to each input modality. The slope of the equations shown in the graph, 0.662 for the phone and 1.249 for the HAT system, highlights the difference in the average learning speed between the two input methods. 
A two-way repeated measures ANOVA was performed on the data with input modality and number of trials as factors. According to Shapiro-Wilk and Mauchly's Test, the assumptions of normality and sphericity, respectively, were not violated for any effect. There was a significant effect of the input modality $\left(\mathrm{F}_{1,7}=12.033, \mathrm{p}=0.010\right)$, but no effect of the number of trials $\left(F_{9,63}=1.676, p=0.114\right)$; the interaction between input method and number of trials was not significant $\left(\mathrm{F}_{9,63}=1.820, \mathrm{p}=0.082\right)$

We expected a statistically significant difference between different trials suggesting the learning effect. However, we empathized the learning effect, especially for HAT, in the User Study 1, where a subject had to repetitively type the same phrase 15 times. Changing the phrase to write everytime, as in this user study, does not help subjects to memorize the movements pattern and correctly write the phrase at hand.

Despite the most recent use of $\mathrm{T} 9$ dates back to at least three years in the past for each user, they all admitted, and it is also confirmed by acquired data, that they easily recalled the lost know-how after a few trials. That significantly helped out in the experiment not only in terms of algorithm confidence, but also at a sensorimotor level: their brain could reuse some previously existing circuits to dexterously coordinate the hands in motion over the phone keyboard. In the neuroscience field, it is well known that learning how to perform a task anew it is rather more complicated and resource consuming than retrieving a long forgotten ability from the long term memory.

\section{Multi-phrase extensive training for a single user}

In order to further investigate the potential impact of HAT, an extensive comparison experiment against a massively spreaded device was conducted with the purpose of identifying the two learning curves. We selected the Nokia 3310 mobile phone and tested its T9 implementation in comparison with HAT. The experiment consisted in writing 10 times each phrase among a set of 10 , randomly chosen as in the previous experiment [11]. The Nokia 3310 was tested first, then the experiment was repeated using HAT (phrases were provided in the same order). Due to the massive number of repetitions, only one volunteer underwent the 200 trials. Although certainly biased subject-wise, we believe that these results are trustworthy and meaningful for the investigation question, i.e., determining the convergence properties of the learning curve as a function of the number of trials.

Figs. 6 and 7 show the results of the comparison experiments in terms of completion time and characters per minute, respectively. Fig. 6a clearly shows a net decrease in the completion time as a function of trials, both in terms of repetitions (horizontal axis) and completed phrases (vertical axis). Although the average completion time is larger (brighter colors toward yellow), the same general trend is evident in Fig. 6b. If compared to the Nokia 3310 case, HAT shows a more significant difference between the first and the last trials. For the sake of completeness we also

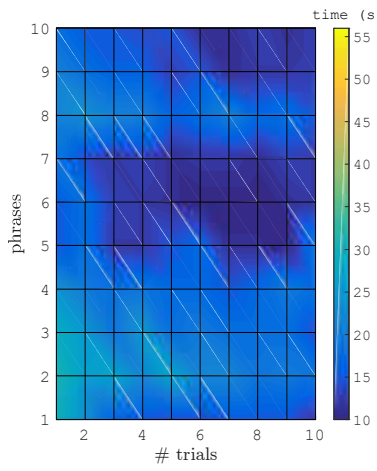

(a) T9 phone

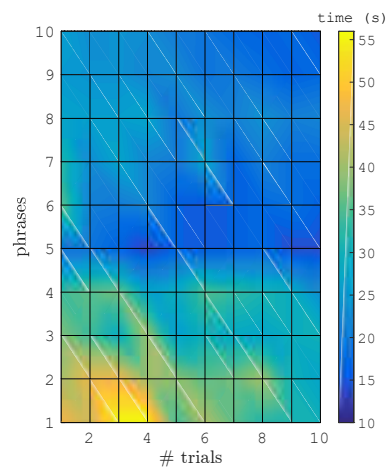

(b) T9 HAT
Fig. 6: Comparison extensive experiment between Nokia 3310 and HAT in T9 Mode: completion time.

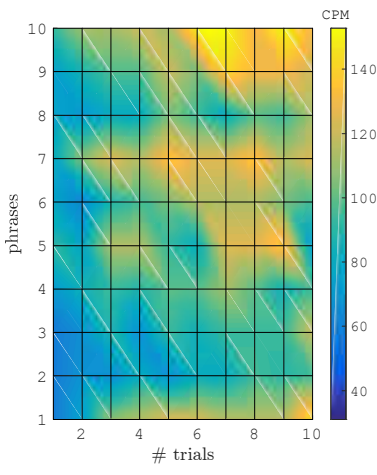

(a) $\mathrm{T} 9$ phone

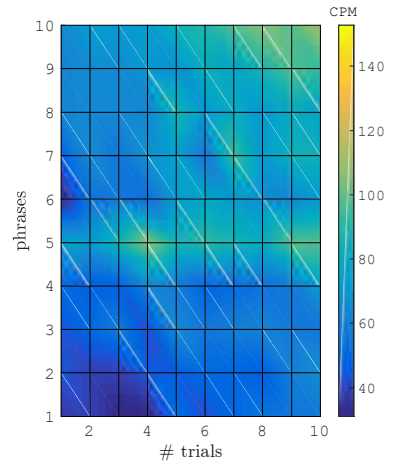

(b) T9 HAT
Fig. 7: Comparison extensive experiment between Nokia 3310 and HAT in T9 Mode: characters per minute.

report Figs. 7a and 7b, in which a similar trend is appreciable in terms of characters per minute.

As a general comment, the figures show that the phone outperforms HAT in absolute value, both in terms of completion time and CPM. However, HAT shows a greater improvement as a function of the number of underwent trials. A possible explanation for this behaviors concerns the a priori knowledge of the tested subject. He, a healthy male aging 32, had no previous experience with that particular model of mobile phone, but owned a similar device for at least 5 years in the past. This established a certain knowledge regarding the sensorimotor coordination of his fingers for the numpad layout, making it fairly straightforward to adapt to the Nokia 3310 . On the other hand, HAT is a completely new input system for which the subject brain couldn't benefit for well established available knowledge. This motivates both the worst absolute performance and the still increasing learning curve at the end of the 100 typed phrases. Additionally, from an informal interview with the subject, he reported a relevant difference between the Nokia and HAT T9 implementations. For the same key combination, the two softwares differ for the order in which possible words are selectable and this has 
a net impact in the completion time. Indeed, when the word is already the right one, the time to scroll the list is saved.

HAT T9 uses the words length as unique sorting criterion: for the same key sequence full words are listed first, then those that need a single additional character and so on. Possible improvements, which will be further investigated in the future, concern words usage frequency and typed sentence analysis for defining a better sorting criterion. On the other hand, the Nokia T9 implementation considers some of the aforementioned criteria, resulting in a more efficient words suggestion.

In order to assess the general mechanical and electrical design of HAT, we asked each subject who participated to the experiments to describe his/her feeling at a glance. Aside from personal comments regarding the potential impact, the great majority of them reported a good degree of reliability, namely occurrences of HAT being unable to properly detect an intentional touch were extremely limited.

\section{CONCLUSIONS AND FUTURE WORK}

This work presents a modular wearable input interface based on finger interaction capable of enabling bluetooth communication with any compatible device, e.g., mobile phones, smartwatches, and smart TVs. Two conductive bands and an accelerometer detect finger tap and orientation, respectively, so that a connected device can be notified. As a case study, we investigated the potential impact of HAT in the field of text input by implementing a smartphone companion application with two available input modalities: multi-tap and a custom T9. HAT was tested in two comparative user studies against a widespread numpad mobile phone (Nokia 3310). For HAT, experimental overall results show an interesting learning slope and a curve fitting study predicts a potential typing speed of at least $60 \mathrm{CPM}$. Bearing in mind the wearable nature of the proposed device, it may not be ideal for writing a novel, but a longer practice can significantly improve the way we currently interact with several portable and non-portable devices.

As mentioned in the experimental evaluation, all the participants who took part in the experiments were not native English speakers. Although this might affect the absolute performance, most of the results presented are not biased because they come from comparisons with other data collected by the same subjects. However, if we consider the potential maximum typing speed depicted in Fig. 4c (using the custom T9 technique), it might be considerably underestimated.

According to results, the performance loss, compared to popular devices having the same typing ability, is acceptable and HAT is a viable way to enable a new era of smart wearable devices. Nowadays, we strongly believe that wearability is of primary importance for input devices.

Besides the case study presented, HAT might be crucial for several different applications ranging from serious games and rehabilitation to its use for visually impaired people.

Although preliminary results seem promising, we need to involve a larger set of subjects to collect more significant data. Moreover, the prototype presented is still far from a fashion accessory; we are currently working on a new design of the input ring in which the form factor will be reduced at least by half and the appearance will play a primary role.

\section{REFERENCES}

[1] T. Lisini Baldi, M. Mohammadi, S. Scheggi, and D. Prattichizzo, "Using inertial and magnetic sensors for hand tracking and rendering in wearable haptics," in Proc. IEEE World Haptics Conference (WHC), 2015, pp. 381-387.

[2] L. K. Simone, N. Sundarrajan, X. Luo, Y. Jia, and D. G. Kamper, "A low cost instrumented glove for extended monitoring and functional hand assessment," Journal of neuroscience methods, vol. 160, no. 2, pp. 335-348, 2007.

[3] L. Dipietro, A. M. Sabatini, and P. Dario, "A survey of glove-based systems and their applications," IEEE Transaction on Systems, Man, and Cybernetics, Part C, vol. 38, no. 4, pp. 461-482, 2008

[4] L. Meli, S. Scheggi, C. Pacchierotti, and D. Prattichizzo, "Wearable haptics and hand tracking via an rgb-d camera for immersive tactile experiences," in Proc. ACM Special Interest Group on Computer Graphics and Interactive Techniques Conference (SIGGRAPH), 2014, p. 56.

[5] S. Scheggi, L. Meli, C. Pacchierotti, and D. Prattichizzo, "Touch the virtual reality: using the leap motion controller for hand tracking and wearable tactile devices for immersive haptic rendering," in $A C M$ SIGGRAPH 2015 Posters. ACM, 2015, p. 31.

[6] L. Jing, Z. Cheng, Y. Zhou, J. Wang, and T. Huang, "Magic ring: A self-contained gesture input device on finger," in Proceedings of the 12th International Conference on Mobile and Ubiquitous Multimedia. ACM, 2013, p. 39.

[7] C. Amma, M. Georgi, and T. Schultz, "Airwriting: a wearable handwriting recognition system," Personal and ubiquitous computing, vol. 18, no. 1, pp. 191-203, 2014.

[8] S. Nirjon, J. Gummeson, D. Gelb, and K.-H. Kim, "Typingring: A wearable ring platform for text input," in Proceedings of the 13th Annual International Conference on Mobile Systems, Applications, and Services. ACM, 2015, pp. 227-239.

[9] C. Kushler, "Aac: Using a reduced keyboard." 1998.

[10] C. James and M. Longé, "Bringing text input beyond the desktop," in CHI Extended Abstracts on Human Factors in Computing Systems, 2000, pp. 49-50.

[11] I. S. MacKenzie and R. W. Soukoreff, "Phrase sets for evaluating text entry techniques," in $\mathrm{CHI}$ 'O3 extended abstracts on Human factors in computing systems. ACM, 2003, pp. 754-755.

[12] P. S. Adler and K. B. Clark, "Behind the learning curve: A sketch of the learning process," Management Science, vol. 37, no. 3, pp. 267281, 1991.

[13] H. R. Thomas, C. T. Mathews, and J. G. Ward, "Learning curve models of construction productivity," Journal of construction engineering and management, vol. 112, no. 2, pp. 245-258, 1986.

[14] W. I. Zangwill and P. B. Kantor, "Toward a theory of continuous improvement and the learning curve," Management Science, vol. 44, no. 7, pp. 910-920, 1998.

[15] H.-S. Park, "Conceptual framework of construction productivity estimation," KSCE Journal of Civil Engineering, vol. 10, no. 5, pp. 311$317,2006$.

[16] H. R. Thomas, "Construction learning curves," Practice Periodical on Structural Design and Construction, vol. 14, no. 1, pp. 14-20, 2009.

[17] J. Han and C. Moraga, "The influence of the sigmoid function parameters on the speed of backpropagation learning," in International Workshop on Artificial Neural Networks, 1995, pp. 195-201. 